\title{
Nanogel loaded with surfactant based nanovesicles for enhanced ocular delivery of acetazolamide
}

This article was published in the following Dove Press journal: International Journal of Nanomedicine

\author{
Rania S Abdel-Rashid (D) \\ Doaa A Helal ${ }^{2}$ \\ Mahmoud M Omar 3,4 \\ Amani M El Sisi ${ }^{5}$ \\ 'Pharmaceutics and Industrial Pharmacy \\ Department, Faculty of Pharmacy, \\ Helwan University, Cairo, Egypt; \\ ${ }^{2}$ Pharmaceutics and Industrial Pharmacy \\ Department, Faculty of Pharmacy, \\ Fayoum University, Fayoum, Egypt; \\ ${ }^{3}$ Pharmaceutics and Industrial Pharmacy \\ Department, Faculty of Pharmacy, Deraya \\ University, El-Minia, Egypt; \\ ${ }^{4}$ Pharmaceutics and Industrial Pharmacy \\ Department, Faculty of Pharmacy, Sohag \\ University, Sohag, Egypt; ${ }^{5}$ Pharmaceutics \\ and Industrial Pharmacy Department, \\ Faculty of Pharmacy, Beni-Suef University, \\ Banī Suwayf, Egypt
}

Objective: Intraocular pressure has always been a great challenge for topical ophthalmic drugs. The study aimed to develop ocular surfactant based nanovesicles (NVs) carried in mucoadhesive nanogel providing efficient topical delivery of acetazolamide (ACZ).

Methods: For the sake of optimizing formulation parameters, the effect of the type of edge activator and its ratio to sorbitan monostearate (Span 60) on the mean particle size, entrapment efficiency (\%EE), and zeta potential (ZP) of produced NVs was investigated.

Results: The selected formulation composed of Span 60:sodium deoxycholate with ratio 80:20 showed an average diameter of $202.90 \mathrm{~nm}, \% \mathrm{EE}$ of $90.2 \%$, and $\mathrm{ZP}$ of $-38.1 \mathrm{mV}$ with a spherical and smooth surface. The ACZ loaded nanovesicles (ACZ-NVs) were embedded in different concentrations of Chitosan-sodium tripolyphosphate (CS-TPP) nanogels. The nanogel prepared using $1.5 \%$ CS showed the most promising viscosity, adhesion time, and rheological behavior (118,246 cP, $290 \mathrm{~min}$, and thixotropic behavior, respectively). The in vitro release of ACZ showed a controlled release profile after incorporation in nanogels. The in vivo irritation test showed minimal irritation for the nanogel formulation compared to ACZ topical suspension. The effect of intraocular pressure lowering was significantly prolonged using ACZ-NV nanogels compared to ACZ oral tablets. Histopathological examination emphasized the healing power of CS on retinal atrophy.

Conclusion: The research work indicated a promising potential for successful topical delivery of ACZ.

Keywords: nanogels, acetazoleamide, intraocular pressure, nanovesicles, edge activators, ocular delivery

\section{Introduction}

Recently, there has been increasing interest in combining the advantages of nanoparticles and hydrogels together, creating nanogels to offer researchers great and novel opportunities for wide varieties of biomedical and pharmaceutical applications. ${ }^{1}$ By modification of a biodegradable nanogel system and fine tuning of its composition, optimal drug loading and controlled release for efficient peroral, rectal, vaginal, ocular, and transdermal drug delivery could be achieved.,3 Moreover, the nanogels can be produced on an industrial scale and the raw materials are easily afforded, providing high economical cost-benefit ratio. ${ }^{4}$ Most importantly, nanogel particles can be rapidly cleared out from the body post treatment via renal excretion and/or enzymatic degradation to reduce the unwanted toxicity. $^{55}$
Correspondence: Rania S Abdel-Rashid Pharmaceutics and Industrial Pharmacy Department, Faculty of Pharmacy, Helwan University, Ain Helwan, Cairo, POB

I 1795, Egypt

Tel +0 020 II5 6995596

Fax +00 2025553487

Email sansoova@yahoo.com 
Efficient ocular drug delivery is one of the puzzling challenges facing pharmaceutical scientists, because of poor bioavailability, weak therapeutic response, and rapid precorneal drug elimination associated with patient compliance problems. ${ }^{6}$ In order to conquer the problems of conventional ocular therapy, neoteric topical delivery systems have been explored by researchers. ${ }^{7,8}$

Acetazolamide (ACZ) is a very well-known carbonic anhydrase inhibitor that is vastly used orally for the diminution of intraocular pressure (IOP) in patients suffering from glaucoma. However, large oral doses of $\mathrm{ACZ}$ are required to obtain the desired lowering in IOP which usually lead to systemic side effects, the most common of which are diuresis and metabolic acidosis. ${ }^{9}$ Most patients are incapable to endure the side effects of ACZ and hence they withdraw from therapy. Thus, a number of scientists sought to develop an effective topical formulation for delivering $\mathrm{ACZ}$ composed of high drug concentration $(2.5-10 \% \mathrm{w} / \mathrm{v})$, viscolizing agents (PVA, HPMC), penetration enhancers (EDTA), and complexing agents (hydroxyl propyl- $\beta$ cyclodextrin) entrapped in particulate drug delivery carriers. ${ }^{10}$ Surprisingly, the complex did not show promising results in terms of the intensity of the decrease in IOP and improvement of side effects. The restrictions in the development of topical formulations of $\mathrm{ACZ}$ are due to its low solubility and inadequate corneal penetration. ${ }^{11}$ Kumar and Rajeshwarrao. ${ }^{12}$ reported that introduction of surfactant based nanovesicles (NVs) has improved both permeability and bioavailability of poorly water soluble drugs. In ocular delivery, NVs also have the ability to prevent metabolism of the drug at the tear/corneal epithelium surface by various enzymes including esterases and oxidoreductases. ${ }^{13}$

Inspired by the unique properties of both nanogels and NVs, this research aimed to develop a drug delivery system for topical administration of $\mathrm{ACZ}$ taking advantage of the high penetration power of surfactant based NVs accompanied with the ease of application and the prolonged residence time of mucoadhesive nanogels. For the sake of optimization, different ratios of Span 60 to different edge activators (Tween 20, Tween 80, sodium deoxycholate) were evaluated for their effect on the physical characterization of NVs. A selected formulation was subsequently introduced into Chitosan polymeric nanogel followed by in vitro and in vivo studies.

\section{Materials and methods}

\section{Materials}

Acetazolamide (ACZ) was kindly received as a gift from CID Pharmaceutical Co., Cairo, Egypt. Sorbitan monostearate (Span 60), polysorbate (Tween 80, Tween 20), sodium deoxycholate (SDC), and Chitosan (low viscosity) were purchased from Sigma-Aldrich Co., St Louis, MO, USA. Sodium tripolyphosphate (TPP) was bought from EVA Cosmetics Co., Cairo, Egypt. Cellulosic membrane Spectra/pore No. 2, 12-14000D was purchased from Spectrum Laboratories, Inc., USA. Ethyl acetate and ethanol were obtained as HPLC grade from El Nasr Pharmaceutical Chemicals Company, Cairo, Egypt.

\section{Methods}

\section{Preparation of ACZ-loaded surfactant based NVs}

ACZ-NVs were prepared by ethanol injection method which was reported as a successful method in previous literature reports. ${ }^{14,15}$ Accurately weighed amounts of ACZ and the different calculated amounts of Span 60 were dissolved in absolute ethanol until clear solutions were obtained. According to the ratios illustrated in Table 1, different types and amounts of edge activators (EAs) (Tween 80, Tween 20, or SDC) were dissolved in distilled water and heated, reaching $60{ }^{\circ} \mathrm{C}$. A glass syringe was charged with the EA solution prepared above and mounted on the infusion pump. The pump was programmed to a flow of $0.5 \mathrm{~mL} \mathrm{~h}^{-1}$, with an inner syringe diameter of $20 \mathrm{~mm}$. The mixture was left at $40^{\circ}$ $\mathrm{C}$ on the magnetic stirrer for $15 \mathrm{~min}$ and another $15 \mathrm{~min}$ at $18{ }^{\circ} \mathrm{C}$. The formulated vesicles appeared as a milky turbid suspension. The composition of the formulated NVs is demonstrated in Table 1.

Table I Composition of acetazolamide nanovesicles

\begin{tabular}{|l|l|l|}
\hline $\begin{array}{l}\text { Formulation (F) } \\
\text { number }\end{array}$ & $\begin{array}{l}\text { Edge activator } \\
\text { (EA) }\end{array}$ & $\begin{array}{l}\text { Ratio of Span } \\
\text { 60:EA }\end{array}$ \\
\hline FI & Tween 80 & $90: 10$ \\
F2 & & $80: 20$ \\
F3 & & $70: 30$ \\
F4 & Sodium & $90: 10$ \\
F5 & deoxycholate & $80: 20$ \\
F6 & & $70: 30$ \\
\hline
\end{tabular}

Abbreviation: Span 60, sorbitan monostearate. 


\section{Characterization of ACZ-NVs}

Determination of percentage drug entrapment efficiency (\%EE)

The $\% \mathrm{EE}$ corresponds to the amount of drug entrapped inside the particles of NVs relative to the total drug content. The \%EE can be determined either directly or indirectly. ${ }^{16,17}$ For the accuracy of procedure results, both methods were applied for determining the concentration of $\mathrm{ACZ}$ in the developed NVs.

\section{Direct determination of \%EE}

The formulated ACZ-NVs were separated by cooling ultracentrifugation at $12,000 \mathrm{rpm}$ and $4{ }^{\circ} \mathrm{C}$ for $15 \mathrm{~min}$. The pellet was dissolved using absolute ethanol and the absorbance was measured spectrophotometrically (JASCO V-530 double beam UV-VIS spectrophotometer connected to a computer loaded with Spectra Manager Program, Japan) at maximum wavelength $265 \mathrm{~nm}$ and then the amount of the ACZ drug was calculated according to the following equation:

$$
\% \mathrm{EE}=\frac{\text { Amount of drug actually present }}{\text { Total amount of drug added }} \times 100
$$

\section{Indirect determination of \%EE}

The concentration of free drug was measured in the aqueous supernatant solution obtained after centrifugation of the NV suspensions. The solutions were measured spectrophotometrically at $265 \mathrm{~nm}$ and the $\% \mathrm{EE}$ was given from the following equation:

$$
\% \mathrm{EE}=\frac{\text { Total amount of drug }- \text { amount of unbound drug }}{\text { Total amount of drug }} \times 100
$$

Determination of particle size, polydispersity index, and zeta potential

The size of vesicles was determined by dynamic light scattering using a Nano ZS Zetasizer (Malvern Instruments, Malvern, UK). A 1-ml sample of nanosuspension was dispersed in $10 \mathrm{ml}$ of double-distilled water. The samples were ultrasonicated for $5 \mathrm{~min}$ prior to size determination. The system was equipped with a $4 \mathrm{~mW}$ helium/ neon laser at $633 \mathrm{~nm}$ wavelength and the sample measured with non-invasive backscatter technology at a detection angle of $173^{\circ}$ using DTS Nano version 6.12 software (Malvern Instruments). All measurements were carried out at $25^{\circ} \mathrm{C}$ assuming $0.8872 \mathrm{cP}$ and 1.330 as the medium viscosity and refractive index, respectively. The average particle size, polydispersity index (PDI), and zeta potential
(ZP) were measured. Triplicate determinations for each formulation were carried out and the mean $\pm \mathrm{SD}$ was deduced. ${ }^{18}$

\section{Transmission electron microscopy}

The morphological examination of the optimum formulation was performed by a transmission electron microscope (TEM) operating at $80 \mathrm{kV}$ (model JEM-1230, JEOL, Tokyo, Japan). One drop of the diluted vesicular dispersion was deposited on the surface of a carbon-coated copper grid, negatively stained with $2 \%$ phosphotungstic acid, and then allowed to dry at room temperature for 10 min for investigation.

\section{Preparation of ACZ-NV loaded nanogel}

The optimized ACZ-NV formulation was incorporated into a topical nanogel according to the method published. ${ }^{19}$ Three stock solutions were prepared by dissolution of the Chitosan (CS) powder at concentrations of $0.8,1.5$, and $2.5 \mathrm{mg} \cdot \mathrm{mL}^{-1}$ in a $2 \%(\mathrm{~m} / \mathrm{v})$ acetic acid aqueous solution and stirred overnight. Nanogels were obtained by an ionotropic gelation process. ${ }^{20}$ An amount of selected ACZ-NV formulation equivalent to $10 \mathrm{mg}$ ACZ was dispersed in the polyanionic phase, that is, TPP $\left(1.2 \mathrm{mg} \cdot \mathrm{mL}^{-1}\right)$ in water $(4.5 \mathrm{~mL})$. The polyanion solution was added dropwise to the CS solution $(9 \mathrm{~mL})$ under sonication ( $750 \mathrm{~W}$, amplitude $32 \%$ ) to obtain stable nanogel. By the end of the addition, magnetic stirring was maintained for $10 \mathrm{~min}^{21}$ The composition of the formulated ACZ-NV nanogels is illustrated in Table 2.

\section{Determination of the gel $\mathrm{pH}$}

A certain amount of the formulated nanogels was dispersed in $30 \mathrm{ml}$ water. The $\mathrm{pH}$ of the various gel formulations was determined using a digital $\mathrm{pH}$ meter (Mettler Toledo MP 220, Switzerland).

Table 2 The physicochemical characterization of acetazolamide loaded nanovesicles

\begin{tabular}{|l|l|l|l|l|}
\hline $\begin{array}{l}\text { Formula (F) } \\
\text { number }\end{array}$ & $\begin{array}{l}\text { Particle } \\
\text { size (nm) }\end{array}$ & PDI & $\begin{array}{l}\text { Zeta poten- } \\
\text { tial (mV) }\end{array}$ & $\begin{array}{l}\% \\
\text { EE }\end{array}$ \\
\hline FI & 446.2 & 0.308 & -20.8 & 58.4 \\
F2 & 301.1 & 0.184 & -19.3 & 67.9 \\
F3 & 218.3 & 0.142 & -18.3 & 59.2 \\
F4 & 224.5 & 0.224 & -34.1 & 76.8 \\
F5 & 202.9 & 0.308 & -38.1 & 90.2 \\
F6 & 194.8 & 0.438 & -41.2 & 81.3 \\
\hline
\end{tabular}

Abbreviations: \%EE, percentage drug entrapment efficiency; PDI, polydispersity index. 


\section{Rheological properties of ACZ-NV loaded nanogel}

The viscosity of ACZ-NV nanogel was measured by a Brookfield Viscometer (Brookfield DV-III Ultra R/S + RHEOMETER, MA, USA). About $0.5 \mathrm{~g}$ of the formulation was applied to the plate and left until the temperature of the cone reached $25 \pm 1{ }^{\circ} \mathrm{C}$. The measurement was started at $20 \mathrm{rpm}$; the speed was gradually increased till it reached $200 \mathrm{rpm}$, and then reduced gradually until reaching the starting rpm. The shear stress versus shear rate measurements were analyzed non-parametrically by calculating the area of hysteresis loops. The linear trapezoidal rule was used to calculate the area under the down and upper curves that were subtracted to get the hysteresis area. To study the flow behavior of the different ACZ-NV loaded nanogels, the log shear rate was plotted against log shear stress and the following equation was applied:

$$
\log \mathrm{G}=\mathrm{NLog} \mathrm{F}-\log \eta
$$

where $\mathrm{G}$ is shear rate $\left(\mathrm{sec}^{-1}\right), \mathrm{F}$ is shear stress $\left(\right.$ dyne $\left./ \mathrm{cm}^{2}\right)$, $\eta$ is viscosity, and $N$ is Farrow's constant. ${ }^{22}$

\section{Determination of the in vitro} mucoadhesive time of ACZ-NV nanogel

The mucoadhesive forces of ACZ-NV loaded nanogels were determined according to the method reported. ${ }^{23}$ Pieces of rabbit ocular tissue were surgically removed and placed in cold saline solution. The tissues were stored frozen in phosphate buffer $\mathrm{pH} 7.4$ and thawed to room temperature before use. At the time of testing, a section of tissue was fixed on the side of a beaker. The gels were attached to the tissues by applying light force with a fingertip for $60 \mathrm{sec}$. The beaker was then filled with $100 \mathrm{~mL}$ phosphate buffer $\mathrm{pH} 6.8$ at $37^{\circ} \mathrm{C}$ and magnetically stirred at $100 \mathrm{rpm}$. The time for complete erosion of the gels from the tissues was taken as an indication of the in vitro adhesion time.

\section{In vitro drug release study}

In vitro release of $\mathrm{ACZ}$ from ACZ-NVs and ACZ-NV nanogel was evaluated. The study was carried out using glass cylindrical tubes opened from both ends with a diameter of $2.5 \mathrm{~cm}$ and tightly covered with a cellophane membrane. One gram of gel was placed in the cylindrical tube; the tube was suspended so that the membrane was just below the surface of $100 \mathrm{~mL}$ phosphate buffer pH 7.4 at approximately $100 \mathrm{rpm}$. Samples of
$1 \mathrm{~mL}$ were withdrawn at predetermined time intervals, and then the release medium was compensated with equal volumes of fresh medium to ensure sink conditions. Samples were filtered through a $0.45-\mathrm{mm}$ pore filter and analyzed for drug content by a UV visible spectrophotometer at $265 \mathrm{~nm}$ after appropriate dilutions. Kinetics of ACZ release was analyzed according to zero order, first order, and Higuchi diffusion models.

\section{Stability study}

Stability of ACZ-NVS

To examine the aggregation of NVs and leakage of ACZ from them during the period of examination, physical stability studies of the prepared NVs were accomplished. The prepared NVs were kept in transparent capped vials (10 mL capacity) at $4{ }^{\circ} \mathrm{C} \pm 1{ }^{\circ} \mathrm{C}$, and $25{ }^{\circ} \mathrm{C} \pm 1{ }^{\circ} \mathrm{C}$ (room temperature) and at $37{ }^{\circ} \mathrm{C}$ for 3 months. Samples from ACZ nanosuspension $(2 \mathrm{~mL})$ were taken periodically. Both the particle size and entrapment efficiency (EE) of the prepared NVs were measured over the stability study period, as already described. Visual inspection was carried out to detect the sedimentation.

\section{Stability of the nanogel formulation}

The prepared ACZ nanogel was stored in amber-colored screw-capped bottles. The bottles were kept at $4{ }^{\circ} \mathrm{C}, 25^{\circ} \mathrm{C}$, and at $37{ }^{\circ} \mathrm{C} / 75 \%$ relative humidity $\pm 5 \%$ for a period of 1 month. Samples of the examined gel $(0.5 \mathrm{~g})$ were withdrawn and evaluated for drug content. The prepared nanogel was examined for any change in appearance at the end of the study. ${ }^{24}$

\section{In vivo study of selected ACZ-NV nanogel}

The Institutional Animal Ethical Committee reviewed the animal protocol prior to the experiment. All rabbits were treated in accordance with the guideline for the care and use of laboratory animals and with the permission of the Faculty of Pharmacy Helwan University Animal Ethical Committee. The study steps were in agreement with ARRIVE guidelines, the Directive of the European Parliament, and the Directive of the European Council 2010/63/EU.

\section{Assessment of ocular irritancy of ACZ-NV nanogel}

In order to evaluate the ocular tolerance of the formulated ACZ-NV nanogel, six male albino New Zealand rabbits 
weighing $2.0-2.5 \mathrm{~kg}$ (2 groups) were used per formulation, observing them for any redness, inflammation, or increased tear production, upon ocular application. A 50 $\mu \mathrm{L}$ aliquot of ACZ-NV nanogel or equivalent concentration of ACZ suspension was instilled in the conjunctival sac of the right eye, while the contralateral eye was kept as control and received no treatment. Both eyes of the rabbits under test were examined using direct visual observation using a slit lamp. ${ }^{15}$

\section{Pharmacodynamic studies}

Adult male albino rabbits were used for in vivo pharmacodynamic studies. The average IOP value for the tested rabbits was $23.9 \pm 1.7 \mathrm{mmHg}$. After $48 \mathrm{~h}$ injection of alpha chymotrypsin in the posterior chamber of the left eye, IOP was increased to above $40 \mathrm{mmHg}$. The IOP of the right eye of each rabbit in all groups maintained its normal value during the study. The 12 rabbits were divided into four groups: one instilled with $50 \mu \mathrm{L}$ ACZ-NV nanogel into the left eye, the second was instilled with $50 \mu \mathrm{L}$ of $\mathrm{ACZ}$ suspension, the third group was treated with an equal amount of ACZ oral tablet (commercial ACZ) in sterile water, while the fourth group was treated with $50 \mu \mathrm{L}$ of physiological saline into the left eye. In order to avoid experimental deviation, the right eye conjunctiva sac (control) was placed in $50 \mu \mathrm{L}$ physiological saline. IOP was measured eight times at the scheduled time intervals $(0$, $0.5,1,2,3,4,5$, and 6 h). Each measurement was repeated for three readings. IOP was measured with a tonometer (Riester, Germany) under surface anesthesia using $0.2 \%$ lidocaine. $^{25}$

\section{Histopathological examination}

The histopathological examination was conducted as a comparative study between the oral commercial tablet of ACZ (Commercial ACZ) and the optimum formulated ACZ-NV loaded nanogel formulation. Six adult male albino rabbits weighing $2-2.5 \mathrm{~kg}$ were divided into two groups. Group I received mucoadhesive ACZ-NV nanogel and group II received an equivalent concentration of Commercial ACZ, for a period of 10 days. The right eye was kept as a control in all experimental rabbits. All of the rabbits were killed after 10 days and their eyes were separated, fixed, cut vertically, dehydrated, cleared, and embedded in paraffin at $60{ }^{\circ} \mathrm{C}$ in a hot air oven for 24 h. Eyes were then sectioned and stained by H\&E. Corneal histological examination was completed after photographing the stained sections using a light microscope. ${ }^{26}$

\section{Statistical analysis}

Pairs of groups were compared by performing one-tailed Student's $t$-test and multiple group comparison was conducted using statistical software (SPSS-11, SPSS Inc., Chicago, IL, USA). All data are presented as mean values with their $\mathrm{SD}$ (mean $\pm \mathrm{SD}$ ). Differences were considered to be statistically significant when the $p$-values were less than 0.05 .

\section{Results and discussion}

\section{Physical characterization of ACZ-NVs Percentage entrapment efficiency}

The first indication of a successful preparation is a reasonable $\% \mathrm{EE}$. All of the formulations exhibited more than $50 \%$ EE which assured the capability of the suggested delivery system to entrap the drug. ${ }^{27}$ Yet there was a significant difference in $\% \mathrm{EE}$ among the formulations ranging from $58.2 \%$ for F3 (Span 60:Tween 20 with ratio $70: 30$ ) to $91 \%$ for F5 (Span 60:SDC with ratio $80: 20$ ) as shown in Table 2. The results showed that a higher ratio of Span 60 showed a significant increasing effect on the \%EE which may be referred to its HLB and transition temperature that qualifies Span 60 to a high EE, which was supported by previous reports. ${ }^{28}$ Another obvious remark was that the type and the concentration of EAs had a significant effect on the \%EE of ACZ-NVs (Table 2). By observing the effect of the EA ratio on $\% \mathrm{EE}$, we can see that the EA ratio of $20 \%$ showed the highest $\% \mathrm{EE}$. However, further increase in the EA ratio will lead to a consequent decrease in $\% \mathrm{EE}$ which may be attributed to pore formation in vesicle bilayers. $^{29}$

\section{Particle size, PDI, and ZP}

The particle size of the NVs is a very critical parameter in order to minimize irritation in ocular tissue, decrease blurry vision, and enhance ocular permeation. ${ }^{30}$ The size of ACZ-NVs ranged between 175.6 and $346.2 \mathrm{~nm}$ as shown in Table 2. The results also showed that only the type of EA has a significant effect on the particle size of NVs, with $p<0.0001$. Results also revealed that the significant decrease in the particle size of ACZ-NVs was dependent on the degree of the hydrophobicity of the EA used. These findings contraindicated the results published by Manosroi et al, ${ }^{31}$ who supposed Tween 80 showed less particle size due to its low HLB compared to SDC.

All formulations showed acceptable PDI where the values ranged from 0.184 to 0.438 as shown in Table 2, indicating homogeneous particle size distribution. The lower the PDI, the higher the uniformity. 
The values of ZP of ACZ-NVs ranged from -20.8 to $-41.2 \mathrm{mV}$ as shown in Table 2 . The negatively charged surface of the NVs is expected to form a shield that prevents the aggregation of the vesicles and maintains stability of the formulation. ${ }^{15}$ It could be clearly seen that ACZ-NVs prepared using SDC showed more negative $\mathrm{ZP}$ values $(-40.1 \mathrm{mV}$ for $30 \%$ $\mathrm{SDC}$ in $\mathrm{F} 6$ ), attributed to their anionic nature, compared to Tween 80 , which is a non-ionic surfactant. ${ }^{32}$ Taking all of the previous results together, the formulation coded F5 was selected for subsequent investigations. The formulation F4 was prepared using Span 60: SDC with ratio 80:20. It showed the highest \%EE (90.2\%), nearly smallest particle size (202.9 $\mathrm{nm})$, a highly accepted value for PDI (0.308), and a high value of $\mathrm{ZP}(-38.1 \mathrm{mV})$. The ACZ-NVs coded F5 were incorporated into a CS-TPP nanogel and subjected to further evaluation.

\section{TEM examination}

The TEM micrographs revealed successful formation of nanovesicular particles with spherical shape and smooth surface. The micrographs are shown in Figure 1.

\section{Physical characterization of ACZ-NV loaded nanogels \\ $\mathrm{pH}$ of nanogels}

The measured $\mathrm{pH}$ of the nanogels ranged from 5.8 to 6.6 which is considered slightly acidic due to the presence of $2 \%$ acetic acid in the preparation. It is very well known that $\mathrm{CS}$ only dissolves in acidic $\mathrm{pH}$, where a weak basic $\mathrm{pH}$ caused by TPP is a must for the ionization of CS and its cross-linking by ionic gelation. ${ }^{33}$ However, the tolerability of eye to this range of $\mathrm{pH}$ was reported as acceptable in various recent research studies. ${ }^{34}$

\section{Rheological studies}

The CS nanogels were widely studied as potential nanocarriers of bioactive molecules in mucous-rich tissues such as ocular and nasal ones. ${ }^{35}$ The effect of different concentrations of CS on the rheological properties of produced nanogels was clearly observed as a significant increase $(p<0.005)$ in the value of viscosity, Farrow's constant, and area under the hysteresis loop followed by a non-significant decrease (plateau) in the previously mentioned parameters (Table 3 ). Their values revealed that all CS-TPP nanogel formulations showed pseudoplastic flow with variable thixotropic behavior which is highly recommended for achieving prolonged residence on
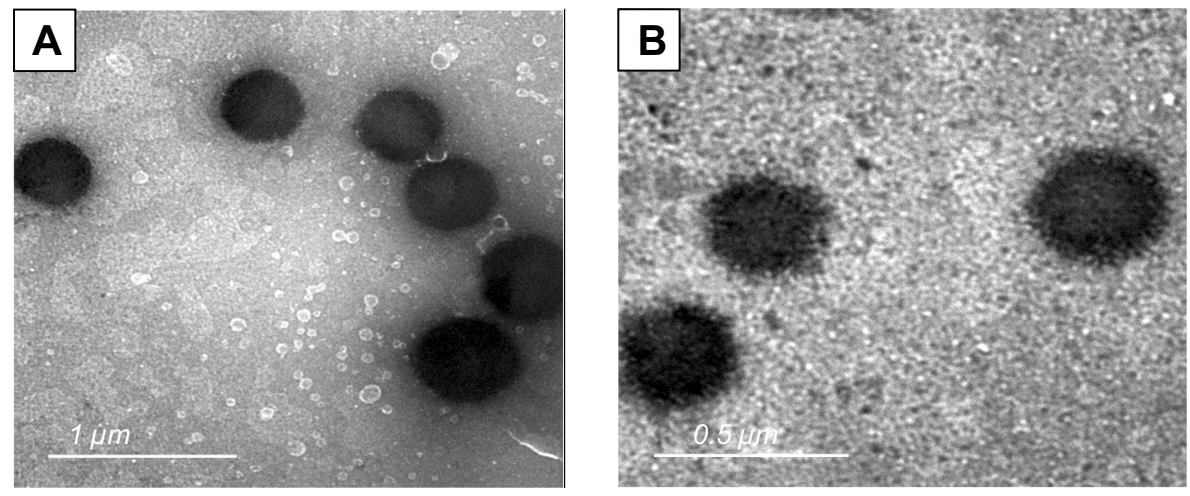

Figure I Transmission electron microscope (TEM) micrographs of prepared acetazolamide loaded nanovesicles. (A) TEM micrograph of F2 (Span 60:Tween 80 ratio 80:20). (B) TEM micrograph of F5 (Span 60:sodium deoxycholate ratio 80:20).

Abbreviations: F, formulation; Span 60 , sorbitan monostearate.

Table 3 Composition and characterization of ACZ-NV nanogels

\begin{tabular}{|l|l|l|l|l|}
\hline $\begin{array}{l}\text { CS concentra- } \\
\text { tion } \\
(\% \mathbf{w} / \mathbf{w})\end{array}$ & $\begin{array}{l}\text { Viscosity (minimum) } \\
(\mathbf{c P})\end{array}$ & $\begin{array}{l}\text { Farrow's constant } \\
\mathbf{( N )}\end{array}$ & $\begin{array}{l}\text { Surface area of hysteresis } \\
\text { loop }\end{array}$ & $\begin{array}{l}\text { Time of adhesion } \\
\text { (min) }\end{array}$ \\
\hline 0.8 & 89,352 & 1.4 & 3198.50 & 160 \\
1.5 & 118,246 & 1.19 & 3983.95 & 290 \\
2.5 & 110,832 & 1.12 & 3854.32 & 282 \\
\hline
\end{tabular}

Note: *All of the gels were loaded with $1 \% \mathrm{w} / \mathrm{w}$ ACZ-NV selected formulation number 5 (F5).

Abbreviations: ACZ-NV, acetazolamide loaded nanovesicle; CS, chitosan. 
the mucous membrane. ${ }^{3}$ The nanogel prepared with $1.5 \% \mathrm{w} /$ w CS showed the best rheological properties. These results could be explained by a high degree of cross-linking because all cross-linking sites at CS polymer are bound to TPP. ${ }^{36}$ Another benefit of increasing viscosity of the CS nanogels is prevention of NVs from leaving the formulation.

\section{Determination of in vitro mucoadhesion time}

The long duration of preocular retention of the formulated ACZ-NV nanogel (Table 3) is based on the bioadhesive power of CS, varying from $160 \mathrm{~min}$ to $290 \mathrm{~min}$. The reinforcement of the mucoadhesive forces could be explained by the fact that secondary bond forming groups (eg hydroxyl, ether oxygen, and amine) are the principal source of mucoadhesion. The cellulosic polymers have an abundance of hydroxyl and ether groups along their length. ${ }^{37}$ All nanogel formulations eroded completely without being detached from the tissues. The high residence time shown by the prepared gels gave ACZ-NVs the chance to be absorbed through the surface of the mucosa and consequently increase the bioavailability. ${ }^{38}$

Based on rheological properties and time of adhesion results, the ACZ-NV loaded ophthalmic nanogel composed of $1.5 \% \mathrm{CS}$ polymer was selected to be subjected for further in vitro and in vivo studies.

\section{In vitro drug release study}

The effect of the drug delivery system on release of ACZ was investigated. The results illustrated in Figure 2 showed a biphasic release pattern for the ACZ-NVs which seemed to be a characteristic of a bilayered vesicle. ${ }^{39}$ Rapid drug leakage was observed during the initial phase where about $12 \%$ of the entrapped ACZ was released from the surfactant based vesicular formulation in the first hour. This could be explained by the fact that ACZ was mainly incorporated between the fatty acid chains in the lipid bilayers of surfactant based NVs which led to rapid release upon dispersing vesicles in buffer until reaching equilibrium. ${ }^{40}$

However, during the following $5 \mathrm{~h}$, only a further $52.9 \%$ of drug was released reaching \%cumulative release of $64.9 \%$. In general, the drug adsorbed on the vesicular surface would be released rapidly.

On the other hand, ACZ release showed a gradual increase and significantly prolonged duration from the loaded CS nanogel (reaching only 38.3\% after $6 \mathrm{~h}$ ). This could be attributed to the influence of viscosity on drug diffusion from mucoadhesive CS nanogel formulation. The increase in formulation viscosity might lead to slow drug diffusion from the gel matrix into the surrounding media. ${ }^{41}$

Kinetic analysis of the release data revealed that ACZ release from both surfactant based NVs and CS nanogels followed a diffusion mechanism (data not shown), where the drug release profile from CS nanogel was more fitted to zero order kinetics.

\section{Stability studies}

The measurements obtained from NVs of the nanosuspension formulation during the stability evaluation are shown in Tables 4 and 5. Insignificant changes $(P>0.05)$ in the EE and vesicle size of the NVs stored at $4{ }^{\circ} \mathrm{C}$ were detected.

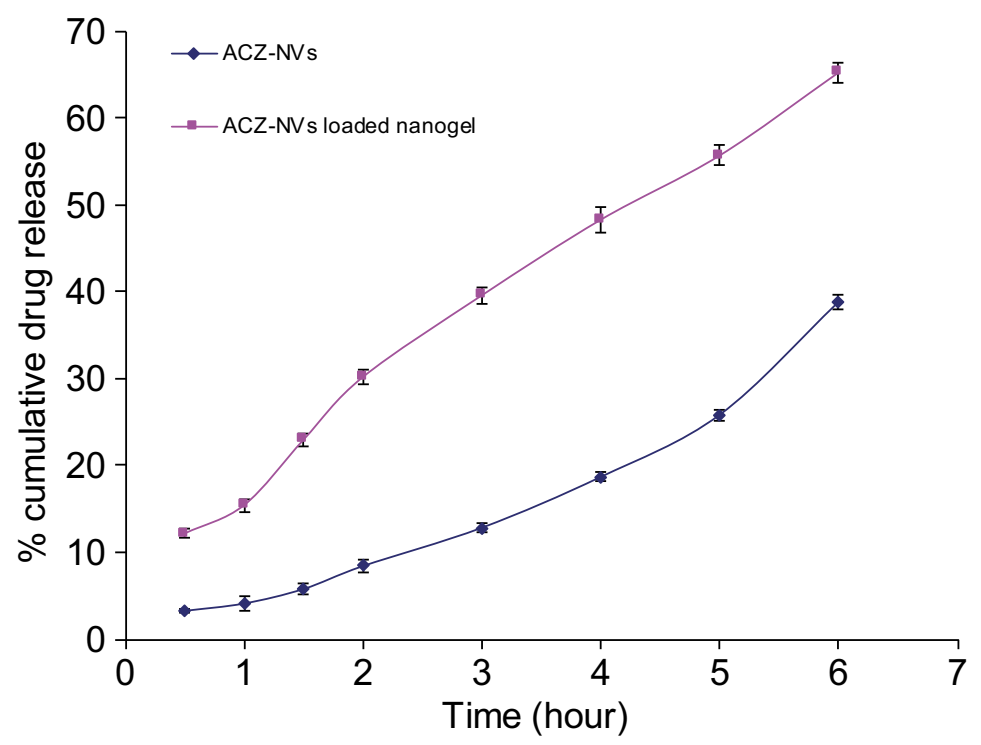

Figure 2 In vitro release profile of acetazolamide from acetazolamide loaded nanovesicles (ACZ-NVs) and ACZ-NVs loaded in Chitosan nanogel. 
Table 4 The entrapment efficiency (EE) measurements (\%) and particle size of the prepared nanovesicles during storage at $4^{\circ} \mathrm{C}$, $25^{\circ} \mathrm{C}$, and $37^{\circ} \mathrm{C}$ over a period of 3 months

\begin{tabular}{|l|l|l|l|l|}
\hline $\begin{array}{l}\text { Examined } \\
\text { characterization }\end{array}$ & Initial & $\mathbf{4}^{\circ} \mathbf{C}$ & $\begin{array}{l}\mathbf{2 5}^{\circ} \\
\mathbf{C}\end{array}$ & $\begin{array}{l}\mathbf{3 7}^{\circ} \\
\mathbf{C}\end{array}$ \\
\hline Particle size (nm) & 202.9 & 214.3 & 272.3 & 523.2 \\
EE (w/w \%) & 90.2 & 88.4 & 80.2 & 78.2 \\
\hline
\end{tabular}

Table 5 The percent drug content (\%) of the prepared nanogel during storage at $4^{\circ} \mathrm{C}, 25^{\circ} \mathrm{C}$, and $40^{\circ} \mathrm{C} / 75 \% \pm 5 \%$ relative humidity over a period of I month

\begin{tabular}{|l|l|l|l|}
\hline \multirow{2}{*}{ Formulation Days } & \multicolumn{3}{|l|}{ Drug content \% } \\
\cline { 2 - 4 } & $\mathbf{4}^{\circ} \mathbf{C}$ & $\mathbf{2 5}^{\circ} \mathbf{C}$ & $\mathbf{3 7}^{\circ} \mathbf{C}$ \\
\hline 0 & 99.9 & 99.9 & 99.9 \\
5 & 99.6 & 99.4 & 98.1 \\
10 & 98.9 & 98.7 & 96.5 \\
15 & 98.4 & 97.8 & 94.3 \\
20 & 97.9 & 96.9 & 91.7 \\
25 & 97.6 & 96.1 & 89.5 \\
30 & 97.1 & 95.4 & 88.9 \\
\hline
\end{tabular}

However, significant changes $(P<0.05)$ in the $\mathrm{EE}$ and particle size of the NVs stored at $25{ }^{\circ} \mathrm{C}$ or $37{ }^{\circ} \mathrm{C}$ were detected. A noteworthy increase in the vesicle size and drug leakage was measured at both $25{ }^{\circ} \mathrm{C}$ and $37{ }^{\circ} \mathrm{C}$ physiologic temperature. Storage of $\mathrm{NVs}$ at $4{ }^{\circ} \mathrm{C}$ has a great effect on their ability to keep a higher percentage of drug, compared to the NVs stored at both $25^{\circ} \mathrm{C}$ and 37 ${ }^{\circ} \mathrm{C}$. Neither color change nor sedimentation was observed regarding $\mathrm{NVs}$ stored at $4{ }^{\circ} \mathrm{C}$. The stability evaluation of the prepared nanogel loaded with NVs represented that insignificant reduction $(P>0.05)$ in the drug content for the nanogel stored at $4{ }^{\circ} \mathrm{C}$ and $25{ }^{\circ} \mathrm{C}$ was detected. A significant decrease $(P<0.05)$ in the drug content for the nanogel stored at $37{ }^{\circ} \mathrm{C}$ was detected, as shown in Table 2. Those results are concurrent with those have found by many scientists. ${ }^{24}$

Stability of the prepared NVs is a critical factor to be considered as a parameter for developing an efficient drug delivery system. The obtained results proved physical stability of the NVs stored at $4{ }^{\circ} \mathrm{C}$ regarding the fusion and aggregation of vesicles. On the contrary, the NVs stored at $25^{\circ} \mathrm{C}$ and $37^{\circ} \mathrm{C}$ showed an increase of vesicle size as a result of coalescence and aggregation of NVs. A significant decrease in EE of the NVs stored at $25^{\circ} \mathrm{C}$ and $37^{\circ} \mathrm{C}$ may be attributed to the developed fluidity of the NVs or possible chemical deterioration of ingredients constituting the NVs. The drug content of the nanogel decreased at a low rate compared to the nanogel stored at elevated temperature. Storage at refrigerator temperature diminishes the problem arising through storage of NVs and nanogel.

\section{In vivo study for ACZ-NV loaded nanogels Ocular irritancy test}

The results showed that over the study period $(6 \mathrm{~h})$ the ACZ-NV nanogel formulation showed no signs of redness, inflammation, or increased tear production compared to the ACZ suspension, proving the safety of the used excipients to be applied topically in the eye. This result was expected based on the small size of the NVs and complete solubility of drug in them. Another explanation is the healing effect of CS on mucous tissue which protected the eye from any possible irritation caused by Span 60 .

\section{Pharmacodynamic study}

For the ACZ suspension treated group, no significant decrease occurred in the IOP as shown in Figure 3. This may be referred to poor absorption and corneal penetration of ACZ through the topical route. For the ACZ-NV treated group, the IOP was decreased to normal values $(22.6 \pm 2.10$ $\mathrm{mmHg}$ ) after $30 \mathrm{~min}$ of administration, the IOP was kept in the normal range for only $2 \mathrm{~h}$, and then the IOP gradually increased. These results were consistent with Hathout et al $^{42}$ who used ACZ in different type of liposomal formulation and studied the IOP lowering effect in normotensive rabbits. Results showed that positively charged multilamellar vesicles of liposomes containing ACZ provide maximum IOP reduction compared to either free drug solution or negatively charged and neutral liposomes. The third group which was treated with ACZ oral tablet (Commercial ACZ) showed a decrease in IOP to normal values after $1.5 \mathrm{~h}$ and the decrement was only sustained for $5 \mathrm{~h}$ followed by a gradual decrease. The most inspiring result was demonstrated in the fourth group treated with ACZ-NV nanogel formulation which showed a gradual decrease in IOP, reaching the normal value after $45 \mathrm{~min}$, and then showed prolonged action to $24 \mathrm{~h}$. This result may be attributed to the mucoadhesive action of the nanogels which maintains the drug in contact with the ocular tissue for a long time. 


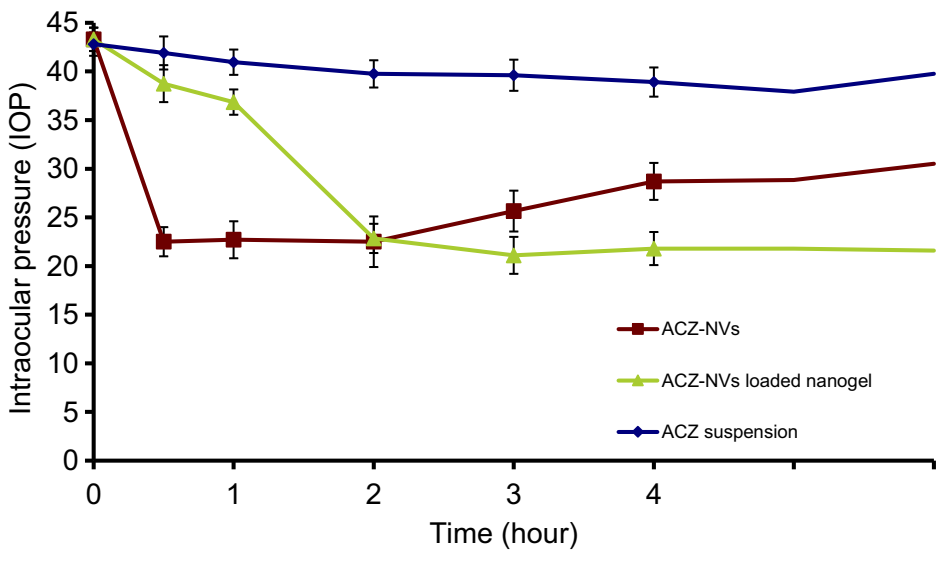

Figure 3 Effect of different formulations of acetazolamide on lowering intraocular pressure.

Abbreviation: ACZ-NV, acetazolamide loaded nanovesicle.

\section{Histopathological examination}

Figure 4A shows the ocular tissues of rabbits with induced glaucoma. Eye lesions of glaucoma induced animals revealed retinal atrophy with loss of retinal ganglion cells and their axons. Decay of a few layers including the inner plexiform layer and nuclear layer was seen. Retinal atrophy was characterized by loss of the internal nuclear layer and ganglion cells. Microcystoid degeneration of the retina of
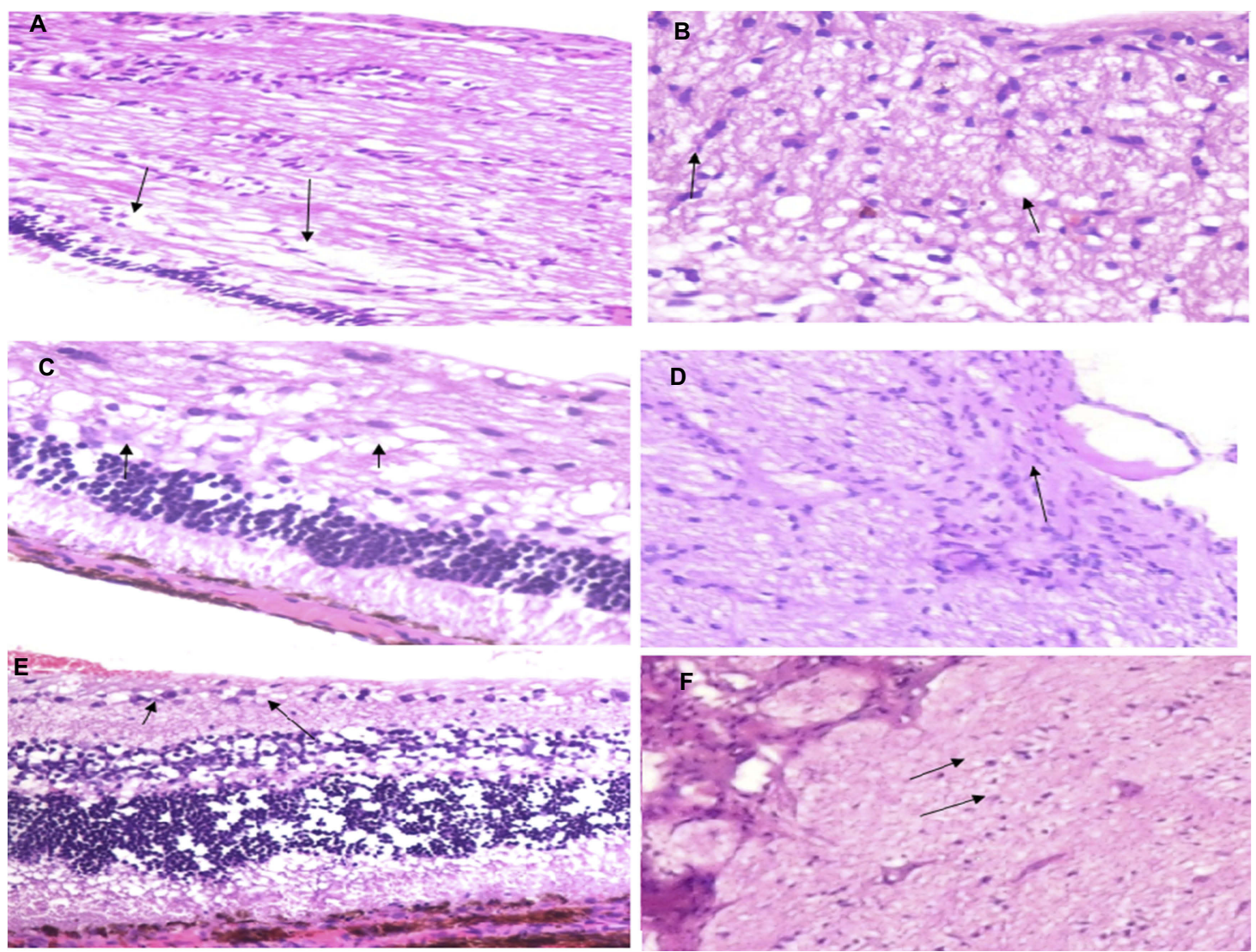

Figure 4 Histopathological examination; findings were appointed with the arrows shown in each image. (A) Glaucoma retina (retina showing loss of internal nuclear layer and ganglion cells with numerous numbers of vacuoles in the choroid). (B) Glaucomatous optic nerve (optic nerve showing thickening, demyelination, and accumulation of fat vacuoles). (C) Glaucoma retina treated with acetazolamide (ACZ) oral tablet (retina showing some loss of inner plexiform layer and ganglion cells with little vacuoles in choroid). (D) Glaucomatous optic nerve treated with ACZ oral tablet (optic nerve showing Wallerian degeneration and minimal loss of myelin sheath). (E) Glaucoma retina treated with ACZ nanovesicles loaded in Chitosan nanogel (retina showing mild swelling of ganglion cells). (F) Glaucomatous optic nerve treated with ACZ nanovesicles loaded in Chitosan nanogel (optic nerve showing mild edema and thickening with remyelination of nerve fibers). Magnification power was I00x. 
glaucomatous eyes was portrayed histologically by various clear round spaces found generally in the inward plexiform layer. ${ }^{43}$ Numerous numbers of vacuoles were typically found in the choroid which went with marked thickening of the retina. The optic nerve of glaucomatous eyes clearly demonstrated Wallerian degeneration in the form of demyelination and accumulation of fat vacuoles in-between the optic fibers, where thickening of the optic nerve was also observed (Figure 4B).

The retina of animal medicated by oral Commercial ACZ tablet demonstrated moderate retinal decay with some loss of inner plexiform layer and ganglion cells. Little vacuoles were found in the choroid which went with slight thickening of the retinal layer (Figure 4C). The optic nerve of glaucomatous eyes treated by oral ACZ demonstrated Wallerian degeneration and minimal loss of the myelin sheath from a great part of the optic nerve filaments compared with the untreated animal group (Figure 4D). These results could be explained by the decrease in IOP produced by oral absorption of ACZ. The retina of glaucomatous eyes treated by ACZNV loaded nanogel showed enhancement of retinal healing with mild swelling of ganglion cells. Healing is a very well established effect of CS hydrogels which may have helped in improving retinal condition. The healing power of CS on the eye is also more prominent due to the small particle size of the nanogel. ${ }^{44}$

No vacuolations of choroid were identified in treated rabbits (Figure 4E). The optic nerve of glaucomatous eyes treated by ACZ-NV loaded nanogel indicated mild edema and thickening synchronized with remyelination of optic nerve fibers (Figure 4F). An absence of any deterioration signs on the retina or optic nerve compared to oral $\mathrm{ACZ}$ proved that the formulated ACZ-NV loaded nanogels used for topically treating glaucoma are safe and efficient.

\section{Conclusion}

There were many attempts to deliver acetazolamide topically with high bioavailability. The administration of ACZ loaded in surfactant based nanovesicles as chitosan nanogels showed promising results. The nanogels showed good mucoadhesion time with the least irritant effect compared to ACZ dispersion. It also showed good lowering power compared to oral $\mathrm{ACZ}$ commercial tablet and ACZ-NVs. The mucoadhesive nanogels are inspiring formulations that are recommended for further applications.

\section{Disclosure}

The authors report no conflicts of interest in this work.

\section{References}

1. Cho H, Gao J, Kwon G. PEG-b-PLA micelles and PLGA-b-PEGb-PLGA sol-gels for drug delivery. $J$ Control Release. 2016;240:191-201. doi:10.1016/j.jconrel.2015.12.015

2. Cho H, Jammalamadaka U, Tappa K. Nanogels for pharmaceutical and biomedical applications and their fabrication using 3D printing technologies. Materials. 2018;11(2):302. doi:10.3390/ma11081451

3. Elkomy M, Elmenshawe S, Eid H, Ali AMA. Topical ketoprofen nanogel: artificial neural network optimization, clustered bootstrap validation, and in vivo activity evaluation based on longitudinal dose response modeling. Drug Deliv. 2016;23(9):3294-3306. doi:10.1080/ 10717544.2016.1176086

4. Neamtu I, Rusu A, Diaconu A, Nita L, Chiriac A. Basic concepts and recent advances in nanogels as carriers for medical applications. Drug Deliv. 2017;24(1):539-557. doi:10.1080/10717544.2016.1276232

5. Sushil K, Arun KI. Biodegradable topical nanogels in the treatment of skin and superficial tumors. Glob J Nano. 2017;1(2):555-556.

6. Kakkar S, Kaur I. Spanlastics - a novel nanovesicular carrier system for ocular delivery. Int $J$ Pharm. 2011;413(1-2):202-210. doi:10.1016/j.ijpharm.2011.04.027

7. Li R, Jiang S, Liu D, et al. A potential new therapeutic system for glaucoma: solid lipid nanoparticles containing methazolamide. J Microencapsul. 2011;28(2):134-141. doi: $10.3109 / 02652048.2010 .539304$

8. Modi K, Shelat PK. Applications of novel vesicular drug delivery system as ocular drug vehicles: a review. Ijpsr. 2012;3(12):4554 4561.

9. Parthibarajan R, Gowrishankar N, Prashanth D, Mallikarjun RV. Formulation and in-vitro evaluation of floating microspheres of acetazolamide. Int J Pharm Pharm Sci. 2011;5(1):447-453.

10. Granero G, Maitre M, Garnero C, Longhi M. Synthesis, characterization and in vitro release studies of a new acetazolamide-HP- $\beta$-CDTEA inclusion complex. Eur J Med Chem. 2008;43(3):464-470. doi:10.1016/j.ejmech.2007.03.037

11. Manchanda S, Sahoo P. Topical delivery of acetazolamide by encapsulating in mucoadhesive nanoparticles. Asian J Pharm Sci. 2017;12 (6):550-557. doi:10.1016/j.ajps.2017.04.005

12. Kumar G, Rajeshwarrao P. Nonionic surfactant vesicular systems for effective drug delivery-an overview. Acta Pharm Sin B. 2011;1 (4):208-219. doi:10.1016/j.apsb.2011.09.002

13. Abraham S, Furtado S, Bharath S, Basavaraj B, Deveswaran R, Madhavan V. Sustained ophthalmic delivery of ofloxacin from an ion-activated in situ gelling system. Pak J Pharm. Sci. 2009;22:2.

14. Basha M, Abd El-Alim S, Shamma R, Awad G. Design and optimization of surfactant-based nanovesicles for ocular delivery of clotrimazole. J Liposome Res. 2013;23(3):203-210. doi:10.3109/ 08982104.2013.788025

15. ElMeshad A, Mohsen A. Enhanced corneal permeation and antimycotic activity of itraconazole against Candida albicans via a novel nanosystem vesicle. Drug Deliv. 2016;23(7):2115-2123. doi:10.3109/ 10717544.2014.942811

16. Salem H, Ahmed S, Omar M. Liposomal flucytosine capped with gold nanoparticle formulations for improved ocular delivery. Drug Des Devel Ther. 2016;10:277. doi:10.2147/DDDT.S91730

17. Elazreg R, Soliman M, Mansour S, El Shamy A. Preparation and evaluation of mucoadhesive gellan gum in-situ gels for the ocular delivery of carbonic anhydrase inhibitor nanovesicles. Ijpsr. 2015;6 (9):3761-3774.

18. Gheran C, Rigaux G, Callewaert M, et al. Biocompatibility of Gd-loaded chitosan-hyaluronic acid nanogels as contrast agents for magnetic resonance cancer imaging. Nanomaterials. 2018;8(4):201. doi:10.3390/nano8040201 
19. Callewaert M, Roullin V, Cadiou C, et al. Tuning the composition of biocompatible Gd nanohydrogels to achieve hypersensitive dual T 1/ T 2 MRI contrast agents. J Mat Chem B. 2014;2(37):6397-6405. doi:10.1039/C4TB00783B

20. El-Menshawe SF, Kharshom R, El Sisi A. Preparation and optimization of buccal propranolol hydrochloride nanoethosomal gel: a novel approach for enhancement of bioavailability. $J$ Nanomed Nanotechnol. 2017;8(2):1000435.

21. Başaran E, Yazan Y. Ocular application of chitosan. Expert Opin Drug Deliv. 2012;9(6):701-712. doi:10.1517/17425247.2012. 681775

22. El-Leithy E, Shaker D, Ghorab M, Abdel-Rashid R. Evaluation of mucoadhesive hydrogels loaded with diclofenac sodium-chitosan microspheres for rectal administration. AAPS PharmSciTech. 2010;11(4):1695-1702. doi:10.1208/s12249-010-9544-3

23. El-Samaligy M, Yahia S, Basalious E. Formulation and evaluation of diclofenac sodium buccoadhesive discs. Int J Pharm. 2004;286(1-2):27-39. doi:10.1016/j.ijpharm.2004.07.033

24. Omar MM, Hassan OA, Elsisi AM. Preparation and optimization of lidocaine transferosomal gel containing permeation enhancers: a promising approach for enhancement of skin permeation. Int J Nanomed. 2019;14:1551-1562. doi:10.2147/IJN.S201356

25. Hassan D, Abdelmonem R, Abdellatif M. Formulation and characterization of carvedilol leciplex for glaucoma treatment: in-vitro, ex-vivo and in-vivo study. Pharmaceutics. 2012;10(4):197. doi:10.3390/pharmaceutics10040197

26. Bancroft J, Stevens A. Theory and Practice of Histological Techniques. 4th ed. Edinburgh: Churchill Livingstone; 1990.

27. Piplani P, Kumar P, Rohilla A, Singla S, Kaur I. Development and evaluation of an ocular niosomal delivery system for some newly synthesized Beta blockers. Ajbps. 2015;5(40):8. doi:10.15272/ajbps. v4i40.634

28. Abdelkader H, Alani AW, Alany R. Recent advances in non-ionic surfactant vesicles (niosomes): self-assembly, fabrication, characterization, drug delivery applications and limitations. Drug Deliv. 2014;21(2):87-100. doi:10.3109/10717544.2013.838077

29. Van Den Bergh BA, Wertz P, Junginger H, Bouwstra J. Elasticity of vesicles assessed by electron spin resonance, electron microscopy and extrusion measurements. Int J Pharm. 2001;217(1-2):13-24.

30. Souto E, Müller R. Investigation of the factors influencing the incorporation of clotrimazole in SLN and NLC prepared by hot high-pressure homogenization. $J$ Microencapsul. 2006;23 (4):377-388. doi:10.1080/02652040500435295

31. Manosroi A, Khanrin P, Werner R, Götz F, Manosroi W, Manosroi J. Entrapment enhancement of peptide drugs in niosomes. J Microencapsul. 2010;27(3):272-280. doi:10.3109/02652040903131293
32. Lee E, Kim A, Oh Y-K, Kim C. Effect of edge activators on the formation and transfection efficiency of ultradeformable liposomes. Biomaterials. 2005;26(2):205-210. doi:10.1016/j.biomaterials.2004.02.020

33. Cho I, Park C, Huh B, et al. Thermosensitive hexanoyl glycol chitosan-based ocular delivery system for glaucoma therapy. Acta Biomater. 2016;39:124-132. doi:10.1016/j.actbio.2016.05.011

34. Liu L, Gao Q, Lu X, Zhou H. In situ forming hydrogels based on chitosan for drug delivery and tissue regeneration. Asian J Pharm Sci. 2016;11(6):673-683. doi:10.1016/j.ajps.2016.07.001

35. Kalam M, Khan A, Khan S, Almalik A, Alshamsan A. Optimizing indomethacin-loaded chitosan nanoparticle size, encapsulation, and release using Box-Behnken experimental design. Int. J. Biol. Macromol. 2016;87:329-340. doi:10.1016/j.ijbiomac.2016.02.033

36. Bhumkar D, Pokharkar V. Studies on effect of $\mathrm{pH}$ on cross-linking of chitosan with sodium tripolyphosphate: a technical note. AAPS PharmSciTech. 2006;7(2):E138-E143. doi:10.1208/pt070250

37. Park C, Kim M, Park M, et al. Nanostructured mucoadhesive microparticles for enhanced preocular retention. Acta Biomater. 2014;10 (1):77-86. doi:10.1016/j.actbio.2013.08.026

38. Ding D, Kundukad B, Somasundar A, Vijayan S, Khan S, Doyle P. Design of mucoadhesive PLGA microparticles for ocular drug delivery. ACS Appl Bio Mater. 2018;1(3):561-571. doi:10.1021/acsabm.8b00041

39. Kaur I, Rana C, Singh M, Bhushan S, Singh H, Kakkar S. Development and evaluation of novel surfactant-based elastic vesicular system for ocular delivery of fluconazole. J Ocul Pharmacol Ther. 2012;28(5):484-496. doi:10.1089/jop.2011.0176

40. Abdelkader H, Ismail S, Kamal A, Alany R. Design and evaluation of controlled-release niosomes and discomes for naltrexone hydrochloride ocular delivery. J Pharm Sci. 2011;100(5):1833-1846. doi:10.1002/jps.22422

41. Rupenthal I, Green C, Alany R. Comparison of ion-activated in situ gelling systems for ocular drug delivery. Part 1: physicochemical characterisation and in vitro release. Int J Pharm. 2011;411(1-2):6977. doi:10.1016/j.ijpharm.2011.03.042

42. Hathout R, Mansour S, Mortada N, Guinedi A. Liposomes as an ocular delivery system for acetazolamide: in vitro and in vivo studies. AAPS PharmSciTech. 2007;8(1):E1-E12. doi:10.1208/pt0801001

43. Tayel S, El-Nabarawi M, Tadros M, Abd-Elsalam W. Promising ion-sensitive in situ ocular nanoemulsion gels of terbinafine hydrochloride: design, in vitro characterization and in vivo estimation of the ocular irritation and drug pharmacokinetics in the aqueous humor of rabbits. Int J Pharm. 2013;443(1-2):293-305. doi:10.1016/j. ijpharm.2012.12.049

44. M Ways T, Lau W, Khutoryanskiy V. Chitosan and its derivatives for application in mucoadhesive drug delivery systems. Polymers. 2018;10(3):267. doi:10.3390/polym10030267
International Journal of Nanomedicine

\section{Publish your work in this journal}

The International Journal of Nanomedicine is an international, peerreviewed journal focusing on the application of nanotechnology in diagnostics, therapeutics, and drug delivery systems throughout the biomedical field. This journal is indexed on PubMed Central, MedLine, CAS, SciSearch ${ }^{\circledR}$, Current Contents $\mathbb{\circledR} /$ Clinical Medicine,
Journal Citation Reports/Science Edition, EMBase, Scopus and the Elsevier Bibliographic databases. The manuscript management system is completely online and includes a very quick and fair peer-review system, which is all easy to use. Visit http://www.dovepress.com/ testimonials.php to read real quotes from published authors. 